\title{
Cárie dentária, alterações de esmalte e necessidades de tratamentto em pré-escolares e escolares de Araras, SP
}

\author{
Dental Caries, enamel defects and treatment needs in children from Araras, SP, Brazil
}

Maria Paula Maciel Rando Meirelles', Elaine Pereira da Silva Tagliaferro², Aline Sampieri Tonello ${ }^{3}$,Silvia Cypriano ${ }^{4}$, Maria da Luz Rosário de Sousa ${ }^{5}$

\section{Resumo}

O objetivo deste estudo transversal foi descrever a prevalência de cárie dentária, hipoplasia, fluorose e opacidade demarcada de esmalte, assim como relatar as necessidades de tratamento em préescolares de 5 anos e de escolares de 12 anos, do município de Araras, em 2004. A amostra probabilística consistiu de 381 indivíduos, sendo 186 pré-escolares de 5 anos e 195 escolares de 12 anos. Os exames epidemiológicos foram realizados por quatro examinadores previamente calibrados, sob luz natural, utilizando-se espelho bucal sonda ball point, seguindo as recomendações da OMS. Cárie dentária foi registrada utilizando-se os índices ceod e CPOD. As lesões sem cavidades ativas, necessidades de tratamento, hipoplasia e opacidade demarcada também foram avaliadas. A fluorose foi registrada seguindo o Índice de Dean nos escolares de 12 anos. Os resultados mostraram que o índice ceod aos 5 anos foi 2,07 ( $d p=3,21)$ e o CPOD aos 12 anos foi de 2,14 ( $d p=2,56)$. Dentre as crianças de 5 e 12 anos examinadas, 52,2\% e 42,3\% estavam livres de cárie, respectivamente. Aproximadamente um terço dos examinados apresentaram sinais de atividade de cárie. As restaurações de uma face foram as necessidades de tratamento predominantes tanto nos pré-escolares $(42,1 \%)$ quanto nos escolares $(39,0 \%)$. A opacidade demarcada esteve presente em $65,1 \%$ dos pré-escolares e $14,4 \%$ dos escolares; a hipoplasia em $5,9 \%$ e $1,5 \%$, respectivamente, e $18 \%$ dos escolares de 12 anos apresentaram fluorose. Pode-se concluir que os pré-escolares e os escolares examinados apresentaram necessidades de baixa complexidade, uma vez que a proporção de livres de cárie foi alta e a atividade da doença foi baixa.

Palavras-chave: Cárie dentária, Necessidades de tratamento, Epidemiologia.

\section{Abstract}

The aim of this cross-sectional study was to describe the prevalence of dental caries and enamel defects, such as hypoplasia, demarcated opacity and fluorosis, as well as to report the treatment needs in 5-year-old pre-schoolchildren and 12-year-old schoolchildren, from Araras, São Paulo State, Brazil, in 2004. The probabilistic sample consisted of 3825 -year-old pre-schoolchildren $(n=186)$ and 12-year-old schoolchildren $(n=196)$. The epidemiological examinations were performed by four calibrated examiners, under natural light, using mirrors and "ball point" probes, following the WHO recommendations. Dental caries was recorded using dmft and DMFT indexes. Fluorosis was recorded following the Dean index in 12-yearolds. The results showed that the dmft index in 5 year-olds was 1.74 $(\mathrm{dp}=3.04)$ and that the DMFT in 12-year-olds was $1.58(\mathrm{dp}=2.39)$. Among the 5- and 12 -year-old children, $52.2 \%$ and $42.3 \%$ were caries free, respectively. Approximately one third of examined children presented signs of caries activity. The one surface filling was the most required treatment in both pre-schoolchildren $(42.1 \%)$ and schoolchildren (39.0\%). The demarcated enamel opacity was detected in $65.4 \%$ of pre-schoolchildren and $14.4 \%$ of schoolchildren and the hypoplasia in $5.9 \%$ and $1.5 \%$, respectively. Regarding fluorosis,
${ }^{1}$ Doutoranda do Programa de Pós-Graduação em Odontologia, Área de Saúde Coletiva da Faculdade de Odontologia de Piracicaba da Universidade Estadual de Campinas.

${ }^{2}$ Doutoranda do Programa de Pós-Graduação em Odontologia, Área de Cariologia da Faculdade de Odontologia de Piracicaba da Universidade Estadual de Campinas.

${ }^{3}$ Doutoranda do Programa de Pós-Graduação em Odontologia, Área de Saúde Coletiva da Faculdade de Odontologia de Piracicaba da Universidade Estadual de Campinas.

${ }^{4}$ Doutoranda do Programa de Pós-Graduação em Odontologia, Área de Saúde Coletiva da Faculdade de Odontologia de Piracicaba da Universidade Estadual de Campinas.

${ }^{5}$ Professora Titular do Departamento de Odontologia Social da Faculdade de Odontologia de Piracicaba da Universidade Estadual de Campinas.

Correspondência: Maria Paula M. Rando Meirelles, Faculdade de Odontologia de Piracicaba - UNICAMP. Departamento de Odontologia Social.

Endereço: Av. Limeira, 901 - Bairro Areião - CEP: 13414-903 - Piracicaba - SP. Email: mariapaula@fop.unicamp.br

$18 \%$ of 12 -year-olds presented enamel disturb. The results indicate that the pre-schoolchildren and schoolchildren examined presented treatment needs of low complexity, since the proportion of caries free was high and the disease activity was low.

Keywords: Dental caries, Treatment needs, Epidemiology.

\section{Introdução e Revisão da literatura}

As condições de sáude bucal de pré-escolares e, principalmente, de escolares têm sido amplamente relatadas na literatura odontológica. Entretanto, grande parte dos trabalhos tem apresentado dados referentes à doença cárie. De fato, por ser o principal problema de saúde pública, esforços têm sido direcionados no sentido de estudar sua epidemiologia. Esta, deve ser utilizada para o conhecimento da distribuição das doenças, conforme preconiza a lei brasileira $\mathrm{n}^{\circ} \mathbf{8 0 8 0}$ de 1990 (BRASIL, 1990), a fim de que medidas apropriadas de controle sejam implementadas.

O primeiro levantamento epidemiológico sobre as condições de saúde bucal dos brasileiros foi realizado em 1986, por iniciativa do Ministério da Saúde (BRASIL, 1988). A média do índice CPOD aos 12 anos foi de 6,8 considerando todo o território nacional. O mais recente levantamento nacional, realizado em 2003 (BRASIL, 2004), mostrou um índice CPOD de 2,7 aos 12 anos, correspondendo a uma redução de 60,3\%, em 17 anos (1986-2003). Os relatos internacionais também vêm evidenciando, inclusive há mais tempo, o declínio da doença cárie (BONECKER; CLEATON-JONES, 2003; MARTHALER, 2004; PAKSHIR, 2004; VAN WYK; VAN WYK, 2004). Os fatores que tem sido relacionados à redução dos níveis da doença são o uso generalizado de fluoretos, o maior acesso aos serviços odontológicos, as mudanças nos critérios de diagnóstico de cárie e a ampliação das ações de promoção e educação em saúde bucal (MARTHALER et al., 1996).

Outras alterações no esmalte dental, como as opacidades fluoróticas e não fluoróticas e as hipoplasias, também deveriam ser estudadas e monitoradas, a fim de se traçar um perfil epidemiológico destas alterações, analisar a tendência de comportamento ao longo do tempo, investigar os fatores causais, assim como o impacto destas alterações em relação a transtornos de sensibilidade e/ou funcionais. 
No levantamento nacional de 2003, apenas a fluorose dentária (opacidade difusa) foi investigada em grupos etários de interesse.

Alguns estudos da literatura têm relatado que alterações no esmalte dentário, como as opacidades e as hipoplasias podem contribuir para uma maior incidência da doença cárie (Monteiro et al., 2003; Ribeiro et al., 2005). A hipoplasia do esmalte, segundo alguns autores pode ser considerada um fator de risco à cárie pelo fato de comprometer a estrutura do esmalte, formando nichos de retenção do biofilme dental, aumentando assim a probabilidade de desenvolvimento da doença (MILGROM., 2000). Devido à diversidade de fatores que afeta o esmalte dentário, os defeitos do esmalte podem ser considerados uma alteração de extrema importância, sendo o ponto inicial de diversos efeitos biológicos formados durante a fase de desenvolvimento do esmalte, podendo ser aplicados em estudos clínicos e epidemiológicos. Assim, considerando que vários estudos demonstram uma relação do defeito de esmalte com o aumento do risco de desenvolver cárie dentária torna-se relevante que esse conhecimento seja estudado para a definição de ações em saúde pública.

Tendo como base a mesma metodologia utilizada no levantamento nacional, o município de Araras, localizado no interior do estado de São Paulo, no ano de 2004, realizou um levantamento epidemiológico sobre saúde bucal de escolares e pré-escolares, pois o município não apresentava dados anteriores passíveis de comparação. Portanto, o objetivo deste estudo transversal foi descrever a prevalência de cárie, fluorose dentária, hipoplasia e opacidades de esmalte, assim como relatar as necessidades de tratamento em préescolares de 5 anos e de escolares de 12 anos do município de Araras, no ano de 2004.

\section{Materiais e Métodos}

\section{Características do município}

O município de Araras pertence à Direção Regional de Saúde - Piracicaba (DIR XV), apresenta população estimada em 115.655 habitantes, uma taxa de urbanização de $94,9 \%$ e o Índice de Desenvolvimento Humano Municipal (IDHM) é de 0,828 (FUNDAÇÃO SISTEMA ESTADUAL DE ANÁLISE DE DADOS, 2005). A fluoretação das águas de abastecimento público foi iniciada em 1970 (Secretaria Municipal de Saúde) e dados divulgados pela Secretaria de Estado da Saúde de São Paulo, no período de 2000 a 2004 demonstraram uma variabilidade para o parâmetro Flúor (0,6 a 0,8 ppm Flúor) de $27 \%$ a $83 \%$ do percentual de amostras com padrões aceitáveis.

\section{Aspectos éticos}

Este estudo foi conduzido após aprovação do Comitê de Ética em Pesquisa da Faculdade de Odontologia de Piracicaba, Processo $\mathrm{n}^{\circ}$ 155/04.

\section{Amostra}

A amostra foi selecionada para o estudo em dois estágios utilizando-se a técnica de amostragem aleatória simples, de forma que as escolas foram selecionadas inicialmente, seguida pela seleção dos pré-escolares e escolares. O tamanho amostral foi calculado considerando a experiência de cárie moderada, uma vez que o município não dispunha de dados anteriores. Considerando que a amostra seria subdividida em pelo menos dois estratos durante a análise dos dados, optou-se por duplicar o tamanho amostral para cada faixa etária. Assim, um total de 508 alunos matriculados em escolas públicas do município, sendo 221 aos 5 anos e 287 aos 12 anos, foram selecionados mediante sorteio aleatório sistemático. Os critérios para a inclusão no estudo foram a ausência de doenças sistêmicas graves, de aparelho ortodôntico fixo com quatro ou mais bandas ortodônticas e a autorização dos pais para a participação na pesquisa por meio do Termo de Consentimento Livre e Esclarecido. A amostra final consistiu de 186 pré-escolares de 5 anos e 195 escolares de 12 anos.

\section{Exame epidemiológico e índices empregados}

Previamente à coleta de dados, uma equipe composta por quatro cirurgiões-dentistas e quatro anotadores participaram do processo de calibração no qual um examinador experiente em levantamentos epidemiológicos (Gold-Standard) procedeu as etapas de treinamento teórico ( 12 horas) e prático ( 24 horas), totalizando 36 horas de calibração. A concordância intra e inter-examinadores foi aferida pela porcentagem de concordância. A equipe só iniciou a coleta de dados após atingir o nível de $85 \%$ de concordância interexaminadores considerando todas as condições a serem avaliadas.

Os exames epidemiológicos foram realizados sob luz natural, utilizando-se espelho bucal plano $\mathrm{n}^{\circ} \mathbf{5}$ e sonda periodontal (ball point), preconizados para uso em levantamentos epidemiológicos, seguindo as recomendações da OMS (WORLD HELATH ORGANIZATION (WHO), 1997). Durante os exames, o examinador sentava-se à frente do paciente.

Os índices e códigos utilizados também seguiram as recomendações da OMS (WHO, 1997). Cárie dentária foi avaliada segundo dois estágios: a) lesões com cavidades utilizando-se o índice ceod aos 5 anos e CPOD aos 12 anos e b) lesões sem cavidades em atividade, segundo os critérios de Nyvad et al. (1999). Os indivíduos com lesões ativas foram considerados aqueles cujas superfícies dentárias apresentaram-se rugosas, opacas e com aspecto de giz, porém sem cavidades. Além desses sinais, foram considerados a presença de placa bacteriana e/ou gengivite e lesão de cárie aguda (aspecto claro e com descamação).

As necessidades de tratamento também foram registradas para as idades de 5 e 12 anos, segundo os critérios do Projeto SB Brasil, 2003 (BRASIL, 2004). A fluorose dentária foi registrada somente aos 12 anos. O exame foi realizado em todos os dentes, utilizando-se o índice de Dean (FEJERSKOV, 1994). Outras alterações de esmalte como hipoplasia e opacidade demarcada foram avaliadas aos 5 e 12 anos; segundo os critérios da OMS (WHO, 1997).

\section{Análise dos dados}

O software EPI-Info, de domínio público, desenvolvido pelo Centers of Disease Control de Atlanta, EUA (CENTER FOR DISEASE CONTROL AND PREVENTION, 1990/1991) foi utilizado, empregando-se o Programa EPIBUCO para o processamento e a análise dos dados.

\section{Resultados}

A distribuição da amostra segundo gênero e idade está descrita na Tabela 1. A taxa de resposta foi de $84,2 \%$ e $67,9 \%$ para as idades de 5 anos e 12 anos, respectivamente.

Tabela 1. Distribuição dos pre-escolares e escolares examinados segundo idade e gênero, Araras, 2004.

\begin{tabular}{|c|c|c|c|c|c|c|}
\hline \multirow{2}{*}{ Idade } & \multicolumn{4}{|c|}{ ceod/CPOD } & \multirow{2}{*}{$\begin{array}{c}\text { Livre de } \\
\text { cárie }\end{array}$} & \multirow{2}{*}{$\begin{array}{c}\begin{array}{c}\text { Atividade de } \\
\text { cárie (mancha } \\
\text { branca) }\end{array} \\
\%\end{array}$} \\
\hline & $\mathrm{n}$ & $\%$ & média & $d p$ & & \\
\hline 5 anos & 186 & 48,8 & 2,07 & 3,21 & 52,2 & 32,8 \\
\hline 12 anos & 195 & 51,2 & 2,14 & 2,56 & 42,3 & 36,5 \\
\hline
\end{tabular}


A média do índice ceod nos pré-escolares foi 2,07 ( $d p=3,21)$ e do índice CPOD nos escolares foi de $2,14(\mathrm{dp}=2,56)$. Dentre as crianças de 5 e 12 anos examinadas, 52,2\% e 42,3\% estavam livres de cárie (ceod $=0$ e $C P O D=0$ ), respectivamente. Cerca de $32,8 \%$ dos pré-escolares e $36,5 \%$ dos escolares apresentaram lesões em atividade.

As distribuições percentuais dos componentes dos índices ceod e CPOD estão apresentadas na Figura 1. O componente obturado apresentou a maior porcentagem no índice CPOD tanto nos préescolares quanto nos escolares, seguido pelo componente cariado (Figura 1). Quanto às necessidades de tratamento, as restaurações de uma face foram predominantes tanto nos pré-escolares $(42,1 \%)$, quanto nos escolares (39,0\%), conforme ilustra o Gráfico 1.

Figura 1. Distribuição percentual dos índices ceod e CPOD, por idade, Araras, SP, 2004.

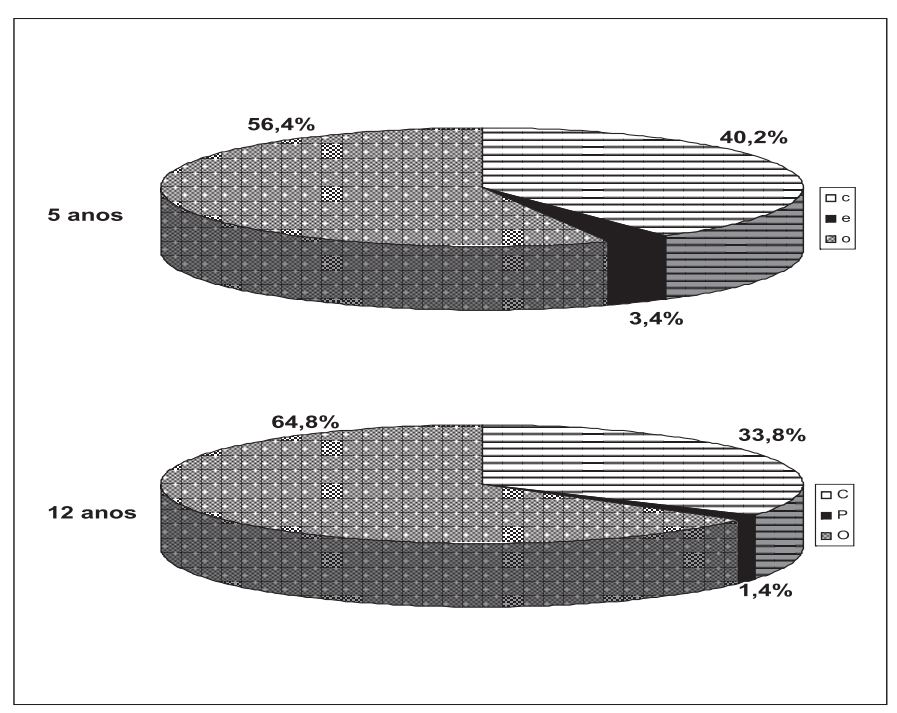

Gráfico 1. Distribuição percentual das necessidades de tratamento, segundo idade, Araras, SP, 2004.

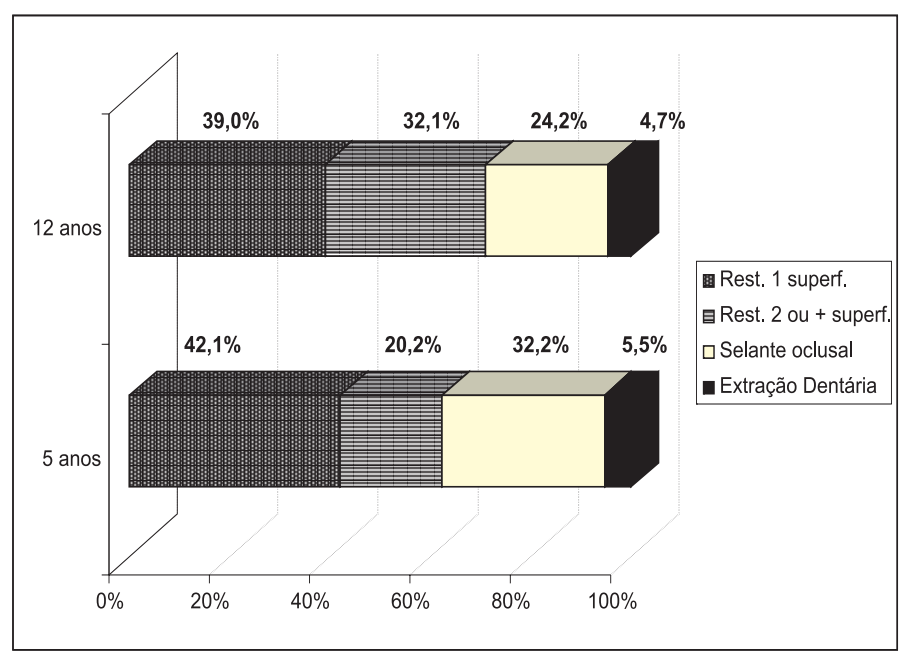

A opacidade demarcada esteve presente em $65,1 \%$ dos préescolares e $14,4 \%$ dos escolares e a hipoplasia em $5,9 \%$ dos pré-escolares e 1,5\% dos escolares. Com relação à fluorose dentária, $82,0 \%$ dos escolares de 12 anos não apresentaram sinais visíveis desta condição (grau 0 e 1, segundo os Índice de Dean). A distribuição percentual dos graus da fluorose dentária está ilustrada no Gráfico 2.
Gráfico 2. Distribuição percentual dos escolares de 12 anos, segundo Índice de Dean, Araras, SP, 2004.

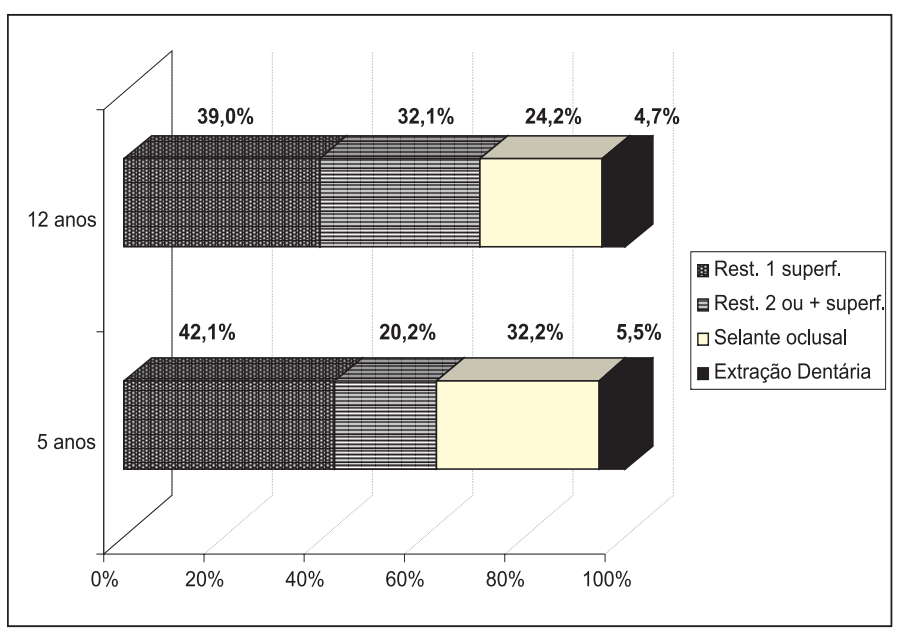

\section{Discussão e Conclusão}

A prevalência de cárie dentária de acordo com Marthaler et al. (1996), vem sofrendo declínio ao longo das últimas décadas e está muito bem documentada na maioria dos países industrializados. Vários fatores têm colaborado com esse processo, tais como os dentifrícios fluoretados, a água fluoretada, os bochechos com flúor, a mudança no consumo de açúcar, a melhoria na limpeza dos dentes, a mudança no diagnóstico da cárie e a melhoria nas condições sócioeconômicas (PINTO, 1992). No entanto, segundo Burt (1998), é princípio básico na prática de saúde pública que se requeira constante observação na experiência de doenças, pois velhos problemas podem ter sua importância diminuída, porém outros novos podem surgir.

O município de Araras não possuía dados epidemiológicos coletados seguindo critérios metodológicos mais acurados. Portanto, os resultados obtidos no presente estudo contribuem com informações relevantes sobre a prevalência e severidade de cárie e fluorose dentária, além de identificam as principais necessidades de tratamento em relação à cárie dentária na população estudada, subsidiando o planejamento local de municípios com níveis semelhantes de doença.

Os dados referentes aos pré-escolares de 5 anos (dentição decídua) demonstraram que a experiência de cárie nessas crianças (ceod=2,07) está um pouco abaixo daquela obtida no levantamento nacional em 2004 tanto para o território nacional (ceod=2,80) quanto para a região Sudeste (ceod=2,50), onde a cidade de Araras está localizada. Além disso, a porcentagem de livres de cárie $(52,2 \%)$ evidencia que a prevalência da doença é menor que a apresentada no território nacional, onde $59,5 \%$ das crianças de 5 anos apresentaram a doença (BRASIL, 2004).

Neste mesmo grupo populacional, considerando os componentes do índice ceod, os dentes restaurados constituíram a maior parte do índice $(56,4 \%)$. Tal resultado merece ser ressaltado, uma vez que se trata de um achado incomum para a faixa etária estudada. Segundo o mais recente levantamento nacional de saúde bucal, o componente obturado tem variado de $4,7 \%$ a $20,0 \%$ do índice ceod, enquanto o componente cariado tem sido o mais prevalente em pré-escolares, variando de $75,6 \%$ a $90,7 \%$ nas regiões brasileiras (BRASIL, 2004). Em Araras, fica evidente, portanto, que a população de pré-escolares tem acesso aos serviços odontológicos assistenciais. Mesmo assim ainda permanece elevado o percentual de dentes cariados $(40,2 \%)$, demonstrando que a demanda por cui- 
dados em saúde bucal ainda é grande, entretanto os procedimentos necessários são de baixa complexidade (restaurações e selantes correspondem a cerca de $95 \%$ das necessidades), podendo ser organizada segundo uma lógica epidemiológica para o controle da doença ou seja, através da somatória de técnicas de mínima intervenção considerando o risco/atividade de doença, procedimentos como tratamento restaurador atraumático (ART), ações preventivas e de promoção de saúde. Além disso, cerca de $32,8 \%$ das crianças apresentaram lesão de cárie em atividade, sugerindo a necessidade de se implantar ou otimizar procedimentos preventivos para essa faixa etária.

Os dados relativos às alterações de esmalte evidenciaram que a opacidade demarcada foi diagnosticada em 14,4\% dos escolares. Estudos foram desenvolvidos com intuito de se avaliar a associação de variáveis clínicas, dentre elas, a presença de defeitos de esmalte, variáveis socioeconômicas, demográficas e a doença cárie (OLIVEIRA et al, 2006). Além disso, os dados apresentados sugerem que a avaliação de defeitos de esmalte seja realizada rotineiramente nos levantamento epidemiológicos, no sentido de monitorar sua tendência.

Com relação à idade de 12 anos, a experiência de cárie dentária encontrada pode ser classificada como baixa, tendo como referência a escala de severidade da OMS (PINTO, 1992), e esteve abaixo dos dados nacionais do levantamento realizado em 2003 $(C P O D=2,78)$, sendo inferior àquela obtida no Estado de São Paulo, cujo CPOD foi de 3,72 em 1998 (NARVAI; CASTELLANOS, 1999) e de 2,33 em 2002.

A porcentagem de livres de cárie (ceod $=0$ e $C P O D=0$ ) nos escolares de Araras foi de $42,3 \%$, sendo inferior à encontrada em Blumenau $(45,3 \%)$ nas escolas públicas (LEITE; RIBEIRO, 2000), porém, superior à encontrada na região de Sorocaba $(32,2 \%)$ (CYPRIANO et al., 2003) e aos resultados do Projeto SB Brasil 2003 em âmbito nacional (31,1\%) (BRASIL, 2004). O componente obturado foi o que apresentou maior percentual dentro do índice CPOD (Figura 1), semelhante aos resultados encontrados em Blumenau (OLIVEIRA; TRAEBERT, 1996) e no Estado de São Paulo (NARVAI; CASTELLANOS, 1999), cujo maior percentual foi relativo a dentes obturados, correspondendo a $64,3 \%$ e $61,4 \%$, respectivamente. Por sua vez, o componente cariado contribuiu com $33,8 \%$ do total do índice CPOD, demonstrando que esses escolares ainda necessitam de acesso aos serviços. Além disso, 36,5\% dos examinados apresentavam atividade de cárie. Nesse sentido, sugere-se que o município elabore políticas públicas ou otimize as já existentes, incluindo procedimentos preventivos e curativos para a idade escolar em programas de saúde bucal.

A maior necessidade de tratamento foi o tratamento restaurador de uma superfície (39,0\%; Gráfico 1), demonstrando que os escolares aos 12 anos necessitam em sua maioria de tratamentos de baixa complexidade. Estudos demonstram que a maior concentração de cárie está em superfícies oclusais de primeiros e segundos molares (HANNIGAN et al., 2000). Cerca de 18,0\% dos escolares apresentaram fluorose dentária (Gráfico 2), resultado superior ao encontrado no ultimo levantamento nacional, cuja prevalência de fluorose foi de $8,56 \%$ considerando todo o território nacional e $13,45 \%$ na região Sudeste (BRASIL, 2004). Os dados referentes a opacidade demarcada e hipoplasia de esmalte demostram que poucos escolares são acometidos por tais alterações.

De acordo com os resultados apresentados, verifica-se que este estudo epidemiológico foi de grande relevância para que se conhecesse a prevalência e atividade de cárie, bem como de fluorose e alterações de esmalte dos pré-escolares e escolares de Araras. De modo geral, os pré-escolares e os escolares examinados apresentaram baixa prevalência de cárie e de defeitos de esmalte, com exceção da opacidade demarcada que foi diagnosticada na maioria dos pré-escolares. Entretanto, considerando que mais que $30 \%$ das crianças examinadas apresentam dentes cariados e atividade de cárie, seria importante sugerir que os serviços odontológicos neste município ofereçam cobertura mais abrangente para estas faixas etárias da população, estabelecendo-se planos de intervenção dentro do contexto da promoção da saúde, monitoramento e manutenção para que se mantenha bons padrões de saúde bucal, com a manutenção funcional dos dentes até faixas etárias mais elevadas (adultos e idosos). Além disso, na prática da saúde se requer constante observação na experiência das doenças, devendo estas serem constantemente monitoradas.

\section{Agradecimentos}

Os autores agradecem à Secretaria Municipal de Saúde de Araras, ao Coordenador de Saúde Bucal, aos Cirurgiões-Dentistas que coletaram os dados do município e a outros profissionais envolvidos neste levantamento epidemiológico, além das pessoas que consentiram em participar deste estudo.

\section{Referências}

BONECKER, M.; CLEATON-JONES, P. Trends in Dental Caries in Latin American and Caribbean 5-6- and 11-13-year-old Children: A Systematic Review. Community Dent. Oral Epidemiol., Copenhagen, v. 31, n. 2, p. 152-157, Apr. 2003.

BRASIL. Lei $n$ ㅇ 8.080, de 19 de setembro de 1990. Dispõe sobre as condições para a promoção, proteção e recuperação da saúde, a organização e o funcionamento dos serviços correspondentes e dá outras providências. Diário Oficial [da] República Federativa do Brasil, Brasília, DF, 20 set. 1990.

BRASIL. Ministério da Saúde. Secretaria Nacional de Programas Especiais de Saúde. Divisão Nacional de Saúde Bucal. Fundação Serviços de Saúde Pública. Levantamento Epidemiológico em Saúde Bucal: Brasil Zona Urbana, 1986. Brasília: Centro de Documentação do Ministério da Saúde, 1988.

BRASIL. Ministério da Saúde. Secretaria de Atenção à Saúde. Departamento de Atenção Básica. Projeto SB Brasil 2003: Condições de Saúde Bucal da população brasileira 2002 - 2003: Resultados Principais. Brasília: Ministério da Saúde, p. 63, 2004.

BURT, B. A. Prevention Policies in the Light of the Changed Distribution of Dental Caries. Acta Odontol. Scand., Oslo, v. 56, n. 3 , p. 179-186, June 1998.

CENTER FOR DISEASE CONTROL AND PREVENTION (CDC). Epi Info Version 5.01. Stone Mountain, 1990/1991.

CYPRIANO,S.; PECHARKI, G. D.; SOUSA, M. L. R.; WADA, R. S. A Saúde Bucal de Escolares Residentes em Locais com ou sem Fluoretação nas Águas de Abastecimento Público na Região de Sorocaba, São Paulo, Brasil. Cad. Saúde Publ., Rio de Janeiro, v.19, n. 4, p.1063-1071, jul./ago. 2003.

FEDERATION DENTAIRE INTERNATIONALE. Global Goals for Oral Health in the year 2000. Int. Dent. J., New York, v. 32, n.1, p.74-77, Mar. 1982

FEJERSKOV, O. Fluorose Dentária: Um Manual para Profissionais da Saúde. São Paulo:Santos, p. 122, 1994.

FUNDAÇÃO SISTEMA ESTADUAL DE ANÁLISE DE DADOS - SEADE. Disponível em: http://www.seade.gov.br/produtos/perfil/perfil.php. Acesso em: 22 abr. 2009. 
GOMES, P.R.; COSTA, S.C.; CYPRIANO, S.; SOUSA, M.L.R. Paulínia, São Paulo, Brasil: Situação da Cárie Dentária com Relação às Metas OMS 2000 e 2010. Cad. Saude Publ., Rio de Janeiro, v. 20, n. 3, p. 866-870, maio/jun. 2004.

HANNIGAN, A. et al. A Caries Susceptibility Classification of Tooth Surfaces by Survival Time. Caries Res., Basel, v. 34, n. 2, p.103-108, Mar./Apr. 2000.

LEITE, I. C.; RIBEIRO, R. A. Dental Caries in the Primary Dentition in Public Nursery School Children in Juiz de Fora, Minas Gerais, Brazil. Cad. Saude Publ., Rio de Janeiro, v. 16, n. 3,p.717-722, July/Sept. 2000.

MARTHALER, T. M.; O'MULLANE, D. M.; VRBIC, V. The Prevalence of Dental Caries in Europe 1990-1995. Caries Res., Basel, v. 30, n. 4, p. 237-255, July/Aug. 1996.

MARTHALER, T. M. Changes in Dental Caries 1953-2003. Caries Res., Basel, v. 38, n. 3, p. 173-181, May/June 2004.

BRASIL. Ministério da Saúde. Disponível em: http://www.saude. sp.gov.br/indicadores/relatorios/municipal/indicador2s/valores.asp Acesso em: 01 ago. 2006.

NARVAI, P.C.; CASTELLANOS, R. A. Levantamento das Condições de Saúde Bucal - Estado de São Paulo, 1998. São Paulo: Núcleo de Estudos e Pesquisas de Sistemas de Saúde, 1999. Relatório apresentado à Secretaria de Estado da Saúde de São Paulo como conclusão do projeto de pesquisa.

NYVAD, B.; MACHIULSKIENE, V.; BAELUM, V. Reliability of a New Caries Diagnostic System Differentiating between Active and Inactive Caries Lesions. Caries Res., Basel, v. 33, n. 4, p. 252-260, July/ Ago. 1999.

OLIVEIRA, J.; TRAEBERT, J. Prevalência de Cárie Dental em Escolares do Município de Blumenau - SC. R. Cienc. Saúde, Campinas, v. 15 , p. 220-236, 1996.

OLIVEIRA, A.F.B.; CHAVES, A.M.B.; ROSENBLATT, A. The Influence of Enamel Defects on the Development of Early Childhood Caries in a Population with Low Socioeconomic Status: a Longitudinal Study. Caries Res., Basel, v. 40, n. 4, p. 296-302, June 2006.

PAKSHIR, H. R. Oral Health in Iran. Int. Dent. J., New York, v. 54, n. 6 , supl. 1, p. 367-372, Dec. 2004.

PINTO, V.G. Financiamento e Organização. In: Saúde Bucal: Odontologia Social e Preventiva . São Paulo: Santos, p. 109-169, 1992.

ROSA, A.G. F; LIA NETO, J.; SERIO, H. B. Avaliação da Assistência Odontológica no Sistema Local de Saúde de São José dos Campos, SP. Divulg. Saúde Debate, Londrina, v. 6, p. 55-60, out. 1991.

VAN WYK, P.J.; VAN WYK, C. Oral Health in South Africa. Int. Dent. J., New York, v. 54, no. 6, supl. 1, p. 373-377, dec. 2004.

VAUGHAN, J.P.; MORROW, R. H. Epidemiologia para os Municípios: Manual para o Gerenciamento dos Distritos Sanitários. 2. ed. São Paulo: Hucitec, p. 180, 1992.

WORD HEALTH ORGANIZATION. Oral Health Surveys: Basic Methods. 4th. ed. Geneve, p. 66, 1997. 